\title{
The Overview of Study on Fatigue Damage Mechanism of Concrete
}

\author{
Lintao Yang ${ }^{1, a}$, Xiaoyan Lan ${ }^{2, b}$ and Feng Guo ${ }^{3, c}$ \\ ${ }^{1}$ East North Road no.6, Jinjiang District, Chengdu, Sichuan Province, China \\ ${ }^{2}$ Baijia County, Liangping Town, Chongqing, China \\ ${ }^{3}$ Yuanshan County, Jiange Town, Gangyuan, Sichuan Province, China \\ a1040982703@qq.com, b1126221684@qq.com, c1182810240@qq.com
}

Keywords: concrete; fatigue; damage; fracture.

Abstract. Concrete materials' damage and destruction under cyclic loading are known as concrete fatigue. Based on the domestic and foreign literature, this paper analyzes and explores the progress and development trend of the fatigue mechanism of concrete on the aspects of experimental study of concrete fatigue, fracture mechanics and damage mechanics research.

\section{Introduction}

Fatigue damage refers to the process of performance degradation of structural materials due to the repeated loading, that means the whole process of the development of the micro-cracks, the formation of macroscopic cracks and the destruction occurs.

Concrete material, a non-uniform multiphase media, are consist of gravel, pebbles, sand, and the cement hydrates crystals and gels which are distributed in disorder in the structure. There are some minor impurities, pores and cracks filled in the concrete body, which constitute the initial damage of the concrete. After being subjected to cyclic loading, the micro-cracks of the concrete will change, expand, connect, and stabilize at a certain level or cracking final destruction in the end.[1] Damage pattern of concrete explained its damage process is actually the expansion and pooling of the small defects, resulting in the process of the reduction of the effective area withstanding stress. Once the damage of a difference, the material constitutive relationship would change. Once the injury tolerance changed, the material constitutive relationship would change.

\section{Concrete Fatigue Damage Mechanism Theory}

Recalling the history of research on fatigue mechanism of concrete, you can find three levels of progressive thread: research based on experiment, research based on fracture mechanics and research based on damage mechanics.

\section{Concrete Fatigue Damage Research based on experiment}

Experimental study on fatigue properties of concrete focus on the S-N curve, fatigue strength, fatigue deformation and fatigue deformation modulus and so on. S-N curve,known as Wohler curve, is to study the relationship between stress levels and fatigue life. Fatigue strength usually expressed as a percentage of its static strength, which means the fatigue stress level with a given fatigue life when fatigue damage occurs.

In experimental studies field, Holmen's research is more representative[2].He systematically studied the deformation performance of the concrete specimen of size $\varphi 100 \mathrm{~mm} \times 250 \mathrm{~mm}$ under amplitude pressure and variable amplitude fatigue loading. His research gives solution to the engineering problems about the fatigue life of concrete under compressive fatigue loads and the irreversible strain development. The subsequent researchers also do a lot of research related to the compressive fatigue of concrete, but generally not exceed the scope of Holmen's. Other researchers take numerous tests on the tension and bending side of concrete fatigue, which is totally different from the compressive fatigue. 


\section{Concrete Fatigue Damage Research Based On Fracture Mechanics}

From a physical perspective, the development of fatigue damage actually corresponds to the development of micro-cracks and micro-defects. Since the tip crack of the quasi-brittle material has a significant non-linear characteristics, therefore, non-linear fracture mechanics become a major tool to study quasi-brittle material fatigue problems.

After Saito[3] analyzed and observed concrete cracks under static tension and fatigue tension through the microscope, he holds the view that fatigue damage is the result out of the extending of the aggregate concrete crack (crack type I), cement mortar cracks (crack type II) and cracks(crack type III) between the aggregate and the mortar. The specific steps are as follow: Crack type III linearly increases with the increase of cyclic loading, type II crack starting from the tip of the type III grows rapidly when reach a certain critical load level type, which makes the concrete lose capacity quickly, and eventually leading to fatigue failure. The proportion of III type crack is very large In the process of destroying, more than about $95 \%$ of the total crack length. It is due to the expanding crack, which leads to the material's damage accumulation in the fatigue process, to stiffness and strength degradation, eventually to the final destruction.

Considering all kinds of nonlinear fracture mechanics model, cohesion crack model (cohe-sive crack model) obtains rapid development and widespread of identity. This model is proposed by Hillerborg at first[4], who applies it to the concrete fatigue crack propagation problems[5]. Hordijk's main achievements include establishing the fatigue crack of $\sigma$-w curve based on cohesion crack model and combining it with the finite element simulation, etc.

\section{Concrete Fatigue Damage Research Based On The Damage Mechanics}

Concrete Fatigue Damage Research Based On The Damage Variable. In order to reflect a lot of material performance degradation caused by the fatigue cracks, damage mechanics gives a probable way to solve the problem. Achievements of the damage mechanics is to combine damage constitutive relation with damage evolution law of research, to analyze the development of material damage under load and the evolution regularity of the constitutive relation and to build materials' continuum damage constitutive equation. In damage mechanics, material's performance degradation caused by the crack propagation under different scales is introduced into the constitutive relation of material through damage variable, which makes the evolution of the damage variable become the core issue of the damage mechanics. Definition methods of damage variable can be more than ten kinds, several representative defined manners are introduced as follows.

(1)Deformation Modulus

According to strain assumption proposed by Lemaitre [6], deformation modulus' attenuation can be used to express the damage variable:

$$
D=1-\frac{E^{2}}{E}
$$

E stands for the initial deformation modulus of concrete; E' stands for the deformation modulus of after a certain cycles.

(2)The Method of Maximum Strain

Zhu [7] makes the conclusion that concrete fatigue maximum strain present three stage development law through concrete under uniaxial and biaxial fatigue test. He thinks that the law is a reflection of internal damage evolution of concrete, and on this basis, he define the damage variable through the maximum strain fatigue :

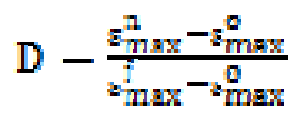


$\varepsilon_{\max }^{\mathrm{n}}, \varepsilon_{\max }^{\mathrm{O}}$ and $\mathrm{E}_{\max }^{\mathrm{f}}$ respectively mean longitudinal initial maximum strain of concrete, maximum strain after fatigue $\mathrm{n}$ times, and the maximum strain.

(3) The Method of residual strain

The relationship between residual strain and cyclic number is similar to that between fatigue cumulative strain and cyclic number, all present three stages character of development relationship, but residual strain is not affected by loading history (or small) when the fatigue damage, suggesting that coagulation on residual strain has its relative independence and therefore residual strain as damage variable has its reliability. The definition of damage variable through residual strain is:

$$
\mathrm{D}=\frac{s_{\mathrm{r}}^{\mathrm{n}}}{\epsilon_{\mathrm{rt}}}
$$

$\mathbf{c}_{\mathrm{r}}^{\mathrm{n}}$ and $\mathbf{c}_{\mathrm{rf}}$ respectively mean residual strain after several times'fatigue and limited residual strain when fatigue damage occurs.

Concrete Fatigue Damage Research Based On thermodynamics. Another basic method of damage mechanics, using the energy conservation principle of the first thermodynamics law and the principle of the constant growth of entropy of the second thermodynamics law. Lemaitre[9] combines strain with damage characteristics and establishs low cycle fatigue damage model of concrete based on the irreversible process theory of thermodynamics in 1985.

Xie [10] studies anisotropy effect of the concrete damage under compression load, and further put forward the isotropic continuum damage model of concrete, induce anisotropic damage model and high voltage load damage model in the continuous medium within the framework of irreversible thermodynamics.

$\mathrm{Ju}$ [11] illustrated the principle of concrete fatigue damage from the field of energy. He considered the essence of concrete fatigue damage was a process of energy transformation and dissipation. If the deformation energy, which accumulating after materials bear load, can't transform to heat energy to scatter in time, it has the only way to dissipate by transforming to surface energy or the others through cracked and destroyed on materials. In the procedure of fatigue damage, due to the high speed of uploading and unloading, the energy accumulated by load working focus to a few concrete native micro-cracks with high endergic and dissipative level; with the increase of cycle index and the energy accumulation, these microcracks continuously extend and gradually evolve to the main cracks, which are decisive to the final damage; with the increase of external load continuous effects, the external work transforms to surface energy to dissipate via absorbed by internal main cracks and supported to extend. When the released energy exceed the energy that the continuously extending cracks need, the extension of internal fatigue cracks shows to the stable extension of internal main cracks. When this extension induce surface visible cracks, the surface fatigue cracks emerge at macroscopic view, leading to fatigue damage soon.

\section{Conclusion}

In this paper, the progress and trends of the concrete fatigue mechanism research were summarized, the review above shows that fatigue research process of concrete includes starting from macro characteristics and gradually going deep into the internal microstructure characteristics; starting from a simple concrete fatigue test at first and gradually going deep into the study of the various factors influencing the fatigue performance of concrete; developing gradually from test and theoretical research to the study of numerical simulation and analysis by software. To sum up, all the research are aimed at making the fatigue test results have a better application to various kinds of actual working conditions. 


\section{References}

[1] HongYi Wang. The overview of concrete Fatigue damage research [J]. Shanxi architecture. (2007), p 18.(in Chinese)

[2] J. Holmen Fatigue of concrete by constant and variable amplitude loading. ACI Special Publication, 75: 71-110, (1982).

[3] M. Saito, Characteristics of micro cracking in concrete under static and repeated tensile Loading [J].Cement and Concrete Research, 17:211-218, (1987).

[4] A. Hillerborg, M. Modeer, PE. Petersson. Analysis of crack formation and crack growth in concrete by means of fracture mechanic sand finite elements. Cement and Concrete Research, 6 (6):773-781, (1976).

[5] D. Hordijk Local approach to fatigue of concrete. [PhD Thesis].Delft: Delft University of Technology, (1991)

[6] J. Lemaitre Application of damage concepts to predict creep-fatigue failure[C] . J. Eng . Mat. Tech, ASME, 101( 1) : 202- 209, (1979).

[7] Jingsong Zhu. Experimental study on fatigue properties of plains concrete under biaxial stress states and theories on failure prediction. Dalian; Dalian University of Science and Technology, (2003).(in Chinese)

[8] Chaoyang Li, Yupu Song, Guofan Zhao. Study on residual strain performance of concrete fatigue. Journal of Dalian University of Technology, ,41(3): 355-358, (2001).(in Chinese)

[9] J. A. Lemaitre. Continuous damage mechanics model for ductile fracture [J] .J. of Eng. Material \& Technology, 107:83-89, (1985).

[10]Heping Xie, Rock, concrete damage mechanics [J].Xuzhou: China University of Mining and Technology press, (1990).(in Chinese)

[11] Yang Ju, Jinglong Pan, ChengMou Fan. A Study on Fatigue Damage Mechanism of Steel Fibre Reinforced Concrete. [J]. Journal of Harbin University of C.E. \& Architecture,30(3):34-40, (1997).(in Chinese) 